\title{
Percepción de Gravedad, Empatía y Disposición a Intervenir en Situaciones de Bullying Físico, Verbal y Relacional en Profesores de $5^{\circ}$ a $8^{\circ}$ Básico
}

\author{
Perception of Severity, Empathy, and Disposition to \\ Intervene in Physical, Verbal, and Relational Bullying \\ Among Teachers From $5^{\circ}$ to $8^{\circ}$ Grade
}

\author{
Verónica Pérez \\ Universidad del Desarrollo
}

\begin{abstract}
Este estudio tuvo como objetivo comparar la percepción, empatía y disposición a intervenir que 88 profesores (23 hombres y 65 mujeres) de $5^{\circ}$ a $8^{\circ}$ básico de colegios particulares de la Región Metropolitana tienen en relación al bullying físico, verbal y relacional. Se adaptó a la realidad chilena un cuestionario con 6 viñetas, desarrollado por Bauman y Del Rio (2006), que describen eventos de bullying físico, verbal y relacional. Se usaron estadísticos descriptivos para caracterizar la muestra y ANOVA mixto para contrastar las hipótesis del estudio. Los profesores calificaron al bullying relacional como el menos grave, presentaron una menor empatía hacia la víctima y estaban menos dispuestos a intervenir que en otros tipos de bullying. Estos resultados son similares a los encontrados en investigaciones realizadas por Yoon y Kerber (2003) y Bauman y Del Rio (2006).
\end{abstract}

Palabras clave: percepción, bullying relacional, maltrato entre escolares, profesores

\begin{abstract}
The purpose of this study was to compare the perception of severity, empathy with the victim, and disposition of 88 teachers ( 23 men and 65 women) between $5^{\text {th }}$ and $8^{\text {th }}$ grade from private schools of the Metropolitan Area of Chile to intervene in bullying incidents, according to the type of bullying: physical, verbal and relational A questionnaire developed by Bauman and Del Rio (2006) was adapted, which includes 6 vignettes that describe physical, verbal, and relational bullying events. Descriptive statistics were used to characterize the sample and mixed ANOVA to test the study hypothesis. The degree of seriousness and teacher involvement was significantly less for relational bullying and presented the lowest level of empathy with the victim. These results are similar to the ones found in the studies of Yoon \& Kerber (2003) and Bauman \& Del Rio (2006).
\end{abstract}

Keywords: perception, relational bullying, peer aggression, teachers

El bullying o matonaje es un tipo de violencia escolar que se distingue por la intención de dañar, por la naturaleza repetitiva de los actos y el desequilibrio de poder entre el bully o agresor y la víctima (Batsche, 2002; Craig, Henderson \& Murphy, 2000; Magendzo, Toledo \& Rosenfeld, 2004; Nicolaides, Toda \& Smith, 2002; Olweus, 1993, 1995; Smith \& Brain, 2000).

Las tasas estimadas de bullying fluctúan entre 15 y $20 \%$ en Australia, Austria, Inglaterra, Finlandia, Alemania, Noruega y Estados Unidos (Veenstra et al., 2005) siendo una de las formas de violencia infantil y juvenil que mayor preocupación ha despertado en los últimos años en Chile y otros países.

Los expertos han definido diversos tipos de bullying, según la naturaleza y el fin de la agresión (Card, Stucky, Sawalani \& Little, 2008). El bullying directo alude a un tipo de agresión física o verbal, en la que el o los agresores enfrentan abierta y directamente a la víctima (Bauman \& Del Rio, 2006; Yoon \& Kerber, 2003). Golpes, empujones y ataques físicos son formas de agresión física, e insultar, fastidiar y amenazar verbalmente son formas de agresión verbal.

Verónica Pérez Villamil, Facultad de Psicología, Universidad del Desarrollo, Santiago, Chile.

La correspondencia relativa a este artículo debe ser dirigida a Verónica Pérez Villamil, El Trovador 4280 Of. 305, Las Condes, Santiago, Chile. E-mail: vperezv@vtr.net 
El bullying indirecto se caracteriza por un tipo de agresión social o relacional, en el que el principal objetivo es dañar la posición y/o aceptación social de la víctima (Bauman \& Del Rio, 2006; Crick, 1996; Crick \& Grotpeter, 1995). Este tipo de agresión se caracteriza por la manipulación deliberada, pero indirecta, de las relaciones interpersonales de la víctima. La exclusión, marginación social, esparcir rumores y aislar serían formas de agresión relacional (Card et al., 2008).

Los límites entre las distintas categorías para clasificar los tipos de agresión son algo difusos. No obstante, existe un consenso en la literatura de incluir las acciones de bullying físico y verbal en la categoría de bullying directo y en considerar al bullying relacional como un tipo de bullying indirecto (Card et al., 2008). Pese a estas distinciones, lo común en los tres tipos de bullying es que la agresión hacia la víctima es sostenida en el tiempo y está en una situación de menor poder que el o los agresores (Bauman \& Del Rio, 2006; Olweus, 1995; Yoon \& Kerber, 2003).

Si bien las agresiones entre pares han existido desde siempre al interior de la escuela, fue Olweus uno de los primeros investigadores en distinguir y conceptualizar el bullying y mostrar el negativo impacto que tiene en la salud mental de los afectados y en el clima escolar (Bauman \& Del Rio, 2006; Magendzo et al., 2004; Olweus, 1993, 1995; Veenstra et al., 2005; Zerón, 2006).

La participación en el bullying, ya sea como agresor o víctima, ha sido asociada a problemas de adaptación social (Cohn \& Canter, 2002): los niños agresores tienen mayor riesgo de verse involucrados en actos delictivos, crímenes y abuso de alcohol (Bauman \& Del Rio, 2006; Olweus, 1993) y los niños victimizados tienen mayor riesgo de presentar depresión y baja autoestima en la adultez (Bauman \& Del Rio, 2006; Kaltiala-Heino, Rimpelä, Rantanen \& Rimpelä, 2000; Olweus, 1993).

Si bien cualquier tipo de bullying afecta la salud mental de los involucrados, el bullying relacional está más fuertemente relacionado con el sufrimiento emocional que el bullying físico y ha resultado ser predictivo de desajustes sociales y psicológicos actuales y futuros (Crick, 1996; Espelage, Mebane \& Swearer, 2004), así como de depresión en la adultez (Crick \& Bigbee, 1998; Olweus, 1993). Los estudios también muestran que para las víctimas de bullying la exclusión social es la peor forma de bullying (Sharp, 1995). Estos hallazgos son preocupantes, ya que los estudios internacionales han mostrado que el bullying relacional despierta menos atención y reacción por parte de los adultos y profesores, existiendo una tendencia por parte de la comunidad educativa a minimizar este tipo de bullying (Mishna, Scarcello, Pepler \& Wiener, 2005).

En un estudio con profesores, Yoon y Kerber (2003) encontraron que las clasificaciones de gravedad y las intervenciones del profesorado fueron significativamente menores para el bullying relacional que para el bullying físico y verbal. Asimismo, el 50\% de las intervenciones frente al bullying físico y verbal involucraba una acción disciplinaria en contra del agresor; en cambio, frente a bullying relacional solo un $10 \%$ lo hacía. Estos hallazgos fueron confirmados por Bauman y Del Rio (2006), quienes replicaron el estudio de Yoon y Kerber (2003) en una muestra de profesores previo a su ingreso al ejercicio profesional. Estos profesores consideraron que el bullying relacional era menos grave que otras formas, presentaron menor empatía con las víctimas del bullying relacional y estaban menos dispuestos a intervenir que en el caso del bullying físico o verbal.

Dependiendo de cómo respondan ante las situaciones de bullying, los profesores pueden tener un rol importante en la prevención y detención de estos hechos. Al estar más cerca de los alumnos y tener mayor presencia en el aula que otros actores del sistema escolar, podrían influir en las conductas futuras de los alumnos víctimas y agresores y mejorar la relación entre los pares (Smith \& Brain, 2000).

En Chile existe escasa literatura que investigue sobre la percepción e importancia que los profesores le asignan a los distintos tipos de bullying, siendo esta una variable central a considerar a la hora de intervenir y prevenir dicha problemática. También son escasas las investigaciones publicadas sobre las intervenciones de los docentes en situaciones de bullying escolar. 
En este contexto, y dada la relevancia de la temática, la presente investigación tuvo como objetivo comparar la percepción de gravedad, la empatía y la disposición a intervenir que profesores que realizan docencia entre $5^{\circ}$ y $8^{\circ}$ básico tienen respecto a los tres tipos de bullying: físico, verbal y relacional.

Tomando como antecedentes los estudios de Bauman y Del Rio (2006) y Yoon y Kerber (2003), se formuló la hipótesis de que los profesores de $5^{\circ}$ a $8^{\circ}$ básico otorgan mayor gravedad y tienen mayor empatía y la disposición a intervenir en situaciones de bullying físico y verbal que en el relacional.

\section{Modelo Ecológico y Violencia Escolar}

Para explicar el fenómeno del bullying existen distintos modelos y teorías, entre estas, las teorías del desarrollo y de la agresión, abordadas desde la epidemiología y psicopatología del desarrollo (Pérez, Fernández, Rodríguez \& De la Barra, 2005), las teorías del apego y mentalización (Lecannelier, 2007), las teorías de la dinámica de grupos, de la psicología social, las teorías del aprendizaje social y de la etología, entre otras.

En esta investigación el bullying será concebido desde el modelo ecológico de Bronfenbrenner (1979), que permite comprender la agresividad entre pares como resultado de procesos de interacción entre variables intrapersonales, interpersonales y grupales, en interacción con el contexto sociocultural donde se desarrollan. Desde este modelo se puede entender que las características personales son producto de las interacciones efectivas y significativas entre personas, objetos y símbolos en un ambiente inmediato en determinado tiempo (Berger \& Lisboa, 2009). Es así como las características a nivel personal en relación con los comportamientos de hostigamiento pueden entenderse como un resultado de las interacciones y relaciones interpersonales de tipo diádico, tales como relaciones tempranas madre y/o cuidador/a-hijo/a y, más tarde, de las relaciones interpersonales que se dan con otros adultos significativos, tales como los profesores y los otros pares en la escuela. Para Bronfenbrenner (1979) todas las relaciones e interacciones, sean estas positivas o negativas, ocurren en determinados contextos y culturas específicas, pudiendo clasificarse en microsistema, mesosistema, ecosistema y exosistema.

El microsistema es el ambiente inmediato donde se desarrollan las relaciones más directas y próximas, en este caso, la escuela, que, dependiendo de cómo cumpla sus funciones, puede detener, prevenir o mantener el circuito de la violencia. En este microsistema es donde se desarrolla la dinámica de la intimidación y donde interactúan los pares entre sí y el profesorado. Desde esta perspectiva, el bullying no se genera solo a partir de las variables individuales del niño que agrede o es agredido, sino también en las interacciones entre las personas y sistemas, en un ambiente inmediato y en una cultura en determinado espacio de tiempo (Berger \& Lisboa, 2009).

El mesosistema considera al conjunto de microsistemas que interactúan entre sí, como la familia y la escuela. En la medida que existe comunicación y contacto entre ambas, más saludable puede ser el desarrollo de los alumnos (Berger \& Lisboa, 2009).

El exosistema incluye los aspectos que no interactúan directamente con el individuo, pero que están presentes e influyen en el desarrollo humano, tal como los valores, la religión y la cultura. En el caso de la violencia, las creencias y percepciones de los sujetos están influidas por los valores y la cultura en la que se desenvuelven. Un ejemplo de ello es que hace un par de décadas los hechos de bullying eran considerados como normales e inofensivos y, en la medida que los valores han ido cambiando y la temática ha sido problematizada en los medios de comunicación, en las políticas sociales y en la cultura, han ido cambiando los significados que las personas le dan a estos hechos (Bonilla \& Oviedo, 2011).

El interés del presente estudio fue profundizar en el microsistema escolar y, específicamente, en el profesor y las percepciones que este tiene en relación a los distintos tipos de bullying. Tal como señala Berger y Lisboa (2009), la posición del profesorado constituye la "línea de fuego" (p. 67) que permite identificar y abordar situaciones de violencia en la escuela. 


\section{Dinámica del Bullying}

En las situaciones de bullying o intimidación escolar participan un conjunto de actores que interactúan entre sí y que algunos autores los han denominado acosadores, víctimas y testigos, identificando ciertas características propias de cada uno de ellos (Sullivan, Cleary \& Sullivan, 2003).

Los acosadores o los bullies han sido caracterizados por su posición de liderazgo y poder, el poco control de la agresión y la necesidad de dominio y control que ejercen hacia otros (Leff, Kupersmidt, Patterson \& Power, 1999).

Distintos autores coinciden en describir a las víctimas de bullying como personas que muestran alguna vulnerabilidad, tales como baja autoestima, tendencia al aislamiento (Leff et al., 1999) y encontrarse en una situación de desventaja emocional, social y/o académica. Al no disponer del apoyo de un grupo, pueden convertirse en una víctima de la intimidación (Sullivan et al., 2003). Asimismo, tienden a pensar en que son las responsables de la intimidación y, por lo tanto, sienten que no pueden hacerle frente, llegando a creer que no sirven para nada y con frecuencia caen en la depresión. En general, una vez que han sido agredidas, están siempre en alerta esperando el próximo ataque.

Finalmente, un tercer actor en la dinámica del bullying son los espectadores, quienes, estando presentes en las situaciones de intimidación, no intervienen directamente o activamente. En la literatura se describen distintos tipos de espectadores: a) los amigos íntimos del acosador que apoyan la intimidación; b) los que se mantienen ajenos e intentan no llamar la atención sobre ellos mismos, pero que, por su aparente neutralidad, parecen tolerar la intimidación y ser inmunes a ella y c) aquellos que se mantienen alejados del intimidador y que pueden abandonar el papel pasivo y convertirse en individuos activos, apoyando a las víctimas y condenando al acosador (Sullivan et al., 2003).

Desde una perspectiva sistémica, los profesores también podrían ser considerados como un tipo de espectador, tal como señalan Newman y Murray (2005), si tienen un rol y participan en dicha dinámica. Cuando las intimidaciones se desarrollan en su presencia, el profesor puede ser un espectador activo o pasivo, dependiendo del rol que asuma. Asimismo, cuando estos hechos ocurren en su ausencia, los alumnos tienden a acudir en su ayuda y el tipo de respuesta que tenga el profesor determinará en alguna medida la mantención o interrupción de la dinámica de bullying (Newman \& Murray, 2005). Desde esta perspectiva, tal como señalan Berger y Lisboa (2009), la posición que el profesor tiene en la situación educativa es de gran importancia para la intervención y prevención de la intimidación.

\section{Percepción de Gravedad, Empatía y Disposición del Profesorado a Intervenir en Situaciones de Bullying}

La percepción de gravedad del bullying, entendida como el grado de seriedad que el profesor le otorga al incidente de bullying que está observando, es una variable que influye en el rol que asume el profesor. Si percibe el hecho como grave, es más probable que asuma un rol activo, interviniendo en la interrupción de la conducta; si no le otorga gravedad no interviene, contribuye a la mantención de estas conductas (Bauman \& del Rio, 2006). En este último caso podría considerarse que asume el rol de un espectador pasivo.

Los profesores tienden a percibir como más serios los hechos de bullying físico y verbal que relacional (Bauman \& Del Rio, 2006; Yoon \& Kerber, 2003). En su estudio Boulton (1997) encontró que la mayoría de los profesores consideraba como bullying las agresiones físicas y amenazas; sin embargo, una proporción importante no consideraba como bullying las conductas intencionales de exclusión y aislamiento. Bauman y Del Rio (2006) plantean este hecho como un problema, ya que los profesores considerarían estas conductas como propias de una edad del desarrollo y/o sin consecuencias para los alumnos, por lo tanto, no intervendrían, perpetuando el circuito de la violencia (Bauman \& Del Rio, 2006; Yoon \& Kerber, 2003).

Mishna et al. (2005) realizaron un estudio con el fin de identificar los factores que influían 
en las respuestas del profesorado ante las situaciones de bullying en el aula. Encontraron que los profesores no intervenían por distintas razones, entre estas, la incertidumbre de cómo responder, no haber sido testigo del incidente e identificar el acoso como un comportamiento propio de la infancia, sin consecuencias graves. Asimismo, los profesores no intervendrían ante los hechos de bullying relacional porque se sentirían con poca capacidad para manejar estos incidentes y al mismo tiempo cumplir los objetivos propios del currículo escolar (Doll, Song \& Siemers, 2004; Mishna et al., 2005).

Otro factor que determina que el profesor responda o no ante los hechos de bullying es que la víctima sea considerada como responsable, es decir, que sus conductas provoquen la agresión en otros. Cuando el profesor percibe que la víctima provoca la agresión, tiende a no intervenir y a no empatizar con ella (Mishna et al., 2005), lo que sería un problema, ya que este tipo de alumno también requeriría de apoyo, acogida y contención (Pérez, 2008).

En síntesis, de acuerdo a los antecedentes antes expuestos, se puede concluir que la dinámica del bullying es compleja y en ella intervienen e inciden múltiples factores y actores, siendo el profesor uno de los actores del microsistema escolar de mayor relevancia para su detección e intervención. Con el fin de aportar a la comprensión del bullying desde la percepción del profesor, en este estudio se buscó comparar la percepción de gravedad, empatía y disposición a intervenir que los docentes tienen en relación a los distintos tipos de bullying.

\section{Método}

\section{Participantes}

Se contactaron cuatro colegios privados de la zona oriente de la Región Metropolitana de Chile, a través del psicólogo u orientador de dicho establecimiento, los que dieron la autorización para realizar el estudio e invitar a los profesores a participar. La muestra estuvo compuesta por 88 profesores, 23 hombres y 65 mujeres. La estrategia de muestreo fue no probabilística intencionada, considerando como criterios de inclusión que fueran profesores jefes o de asignatura que hacían clases en los niveles de $5^{\circ}$ a $8^{\circ}$ básico y con más de un año de trabajo en el establecimiento educacional. Los criterios de exclusión fueron que fueran profesores que estuvieran haciendo práctica profesional y/o que llevasen menos de un año haciendo clases en el lugar de trabajo.

\section{Instrumentos}

Se utilizó el cuestionario reportado por Sheri Bauman y Adrienne Del Rio, de la Universidad del Estado de Arizona (2006). Este instrumento consta de seis viñetas: dos de ellas retratan un escenario de bullying físico (4 y 6), dos describen incidentes de bullying verbal (1 y 3 ) y dos presentan situaciones de bullying relacional ( 2 y 5). Los niños en las viñetas son neutros en cuanto a sexo y etnia-raza. Cada viñeta está seguida por tres ítems, puntuados en una escala Likert de 5 o 4 puntos, que preguntan sobre la percepción del encuestado sobre la gravedad del incidente (rango 1 a 5), el grado de empatía con la víctima (rango 1 a 4) y la probabilidad de intervención en la situación por parte del encuestado (rango 1 a 5). En cada una de las viñetas un mayor puntaje indica mayor nivel de la variable. Los dos últimos ítems son preguntas abiertas que apuntan a cómo respondería el encuestado hacia el agresor y hacia la víctima.

Para el presente estudio este instrumento fue validado a la realidad chilena con la autorización de Bauman y Del Rio (2006, ver Anexo). Para estos efectos se realizó una doble traducción de las viñetas, del inglés al español, por un traductor bilingüe y, luego, un nativo de habla inglesa hizo la traducción del español al inglés para corroborar que la traducción fuese fiel a su origen. Posteriormente, se realizó la validación de contenido a través de cinco jueces externos expertos en la temática de bullying y educación. Se les entregó las seis viñetas junto al cuestionario y se les solicitó que hicieran una revisión de las viñetas en relación a su correspondencia al tipo de bullying que se menciona en cada una, que señalaran si agregarían alguna situación 
nueva (otra viñeta) para cada uno de los tipos de bullying y si cambiarían la forma de expresar la situación (palabras o frases) en cada una de las viñetas correspondientes a los distintos tipos de bullying. Se diseñó una pauta para que completaran la información, en la que también podían agregar, cambiar o modificar algunas de las viñetas y las preguntas del cuestionario. Como criterio para hacer modificaciones se consideró un acuerdo inter-juez de cuatro o más jueces expertos.

Los coeficientes de consistencia interna del instrumento - medida por alfa de Cronbachobtenidos en este estudio fueron 0,74 para la percepción de gravedad, 0,87 para la empatía y 0,54 para la disposición a intervenir, similares a los obtenidos por Bauman y Del Rio (2006; 0,74 para gravedad, 0,85 para empatía y 0,67 para disposición a intervenir).

Las viñetas han sido utilizadas ampliamente en estudios de actitudes y percepción. Pese a que existen cuestionamientos respecto de la validez externa de los resultados, Alexander y Becker (1978) recomiendan esta metodología, argumentando que tiene mayor utilidad que los cuestionarios o entrevistas para evaluar la toma de decisiones y/o conocer cómo los sujetos perciben situaciones en la vida real. Según estos autores y otros, tales como Wilks (2004), las viñetas pueden tener ventajas en controlar los efectos de la deseabilidad social, al poner el problema fuera de la experiencia personal de quien responde, y eliminan los potenciales efectos del observador, ya que se ha visto que la observación de la conducta de los profesores por investigadores puede influir en su comportamiento y podría poner al investigador en una posición difícil si se observaran conductas poco éticas o no profesionales (Gould, 1996). Poulou (2001) plantea que el uso de viñetas es la técnica más apropiada para entender las respuestas cognitivas y afectivas de los profesores a incidentes específicos, proveyendo información esencial para diseñar programas efectivos de entrenamiento.

\section{Procedimiento}

En cada colegio se reunió al grupo de profesores dispuestos a participar en la investigación, los que accedieron voluntariamente. Se les explicó el objetivo del estudio, asegurándoles el carácter anónimo de la información y se les pidió firmar una carta de consentimiento informado. A cada uno se le entregó el cuestionario, el que tomó alrededor de 30 a 40 minutos en ser respondido.

\section{Análisis de la Información}

Se calcularon diversos estadísticos descriptivos (promedio, desviación estándar, gráficos) para caracterizar a la muestra en las variables de interés. Para contrastar las hipótesis se realizó un ANOVA mixto, considerando las mediciones de percepción de gravedad, empatía hacia la víctima y disposición a intervenir para cada categoría de bullying como variable intrasujetos y el sexo de los profesores como variable intersujeto. Para detectar diferencias para cada variable entre los tipos de bullying se observaron los efectos principales de las variables y se realizaron comparaciones por pares, utilizando diferencia de medias con ajuste Bonferroni. Se utilizó la prueba de Mauchly para evaluar el supuesto de esfericidad, utilizando, en el caso de su incumplimiento, el estadístico corrector de Greenhouse-Geisser. Para todos los análisis estadísticos se aceptó una probabilidad máxima de error de tipo I de $\alpha=0,05$.

\section{Resultados}

A nivel muestral, los promedios obtenidos fueron más altos para el bullying físico que para el bullying verbal y relacional en percepción de gravedad, empatía con la víctima y disposición a intervenir. Asimismo, la dispersión de los puntajes fue menor para el bullying físico en cada una de las variables (ver Tabla 1 ). 
Tabla 1

Estadisticos Descriptivos de Percepción de Gravedad, Empatía con la Victima y Disposición a Intervenir por Parte de los Profesores, de Acuerdo a Cada Tipo de Bullying, Según Sexo

\begin{tabular}{lcccccc}
\hline \multirow{2}{*}{\multicolumn{1}{c}{ Variable }} & \multicolumn{2}{c}{ Hombre } & \multicolumn{2}{c}{ Mujer } & \multicolumn{2}{c}{ Total } \\
\cline { 2 - 7 } & $M$ & $D E$ & $M$ & $D E$ & $M$ & $D E$ \\
\hline Percepción de gravedad físico & 4,65 & 0,54 & 4,77 & 0,44 & 4,71 & 0,49 \\
Percepción de gravedad verbal & 4,12 & 0,73 & 4,48 & 0,46 & 4,39 & 0,58 \\
Percepción de gravedad relacional & 3,94 & 0,70 & 4,14 & 0,58 & 4,12 & 0,61 \\
Empatía con la víctima físico & 3,61 & 0,77 & 3,64 & 0,62 & 3,63 & 0,66 \\
Empatía con la víctima verbal & 3,28 & 0,90 & 3,60 & 0,64 & 3,48 & 0,75 \\
Empatía con la víctima relacional & 3,23 & 0,81 & 3,62 & 0,61 & 3,48 & 0,70 \\
Disposición a intervenir físico & 4,94 & 0,22 & 4,95 & 0,19 & 4,95 & 0,20 \\
Disposición a intervenir verbal & 4,80 & 0,35 & 4,89 & 0,23 & 4,86 & 0,27 \\
Disposición a intervenir relacional & 4,75 & 0,33 & 4,68 & 0,50 & 4,70 & 0,46 \\
\hline
\end{tabular}

El supuesto de esfericidad de la matriz varianza-covarianza solo se cumplió para la variable gravedad percibida, por lo que en las variables empatía con la víctima y disposición a intervenir se utilizó el estadístico corrector de Greenhouse-Geisser como criterio de decisión.

El ANOVA mixto arrojó un efecto principal del tipo de bullying en la variable percepción de gravedad, es decir, hubo diferencias significativas en la percepción de gravedad de cada tipo de bullying, $F(2,170)=49,20, p<0,001, \eta^{2}=0,37,95 \%$ ICs $[4,60,4,82],[4,27,4,52]$ y $[3,99,4,27]$ para bullying físico, verbal y relacional, respectivamente. No se encontró un efecto significativo en la interacción con sexo, $F(2,170)=2,11, p=0,125$.

Analizando las comparaciones del efecto principal del tipo de bullying, se encontraron diferencias significativas $(p<0,05)$ entre los puntajes de percepción de gravedad para cada tipo de bullying (ver Figura 1).

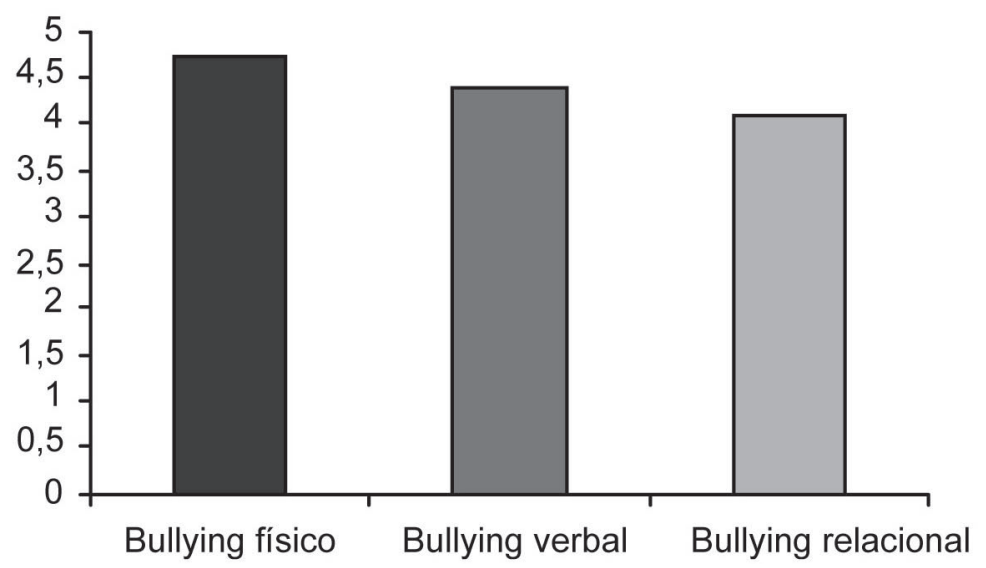

Figura 1. Medias de percepción de gravedad, según tipo de bullying. 
El ANOVA mixto también mostró un efecto principal del tipo de bullying en la variable empatía con la víctima, es decir, hay diferencias significativas en la empatía con la víctima para cada tipo de bullying, $F=4,99, p=0,011, \eta^{2}=0,09,95 \%$ ICs $[3,47,3,77]$, [3,31, 3,65] y $[3,31$, $3,65]$ para bullying físico, verbal y relacional, respectivamente. Además, se detectó un efecto significativo de la interacción con sexo, $F=3,54, p=0,038, \eta^{2}=0,06,95 \%$ ICs $[3,45,3,78]$, [3,24, $3,93],[3,41,3,76],[2,84,3,56],[3,48,3,70]$ y $[2,84,3,48]$ para bullying físico, verbal y relacional en mujeres y hombres, respectivamente. La interacción encontrada se presenta en la Figura 2 y muestra que los hombres empatizaron más con las víctimas de bullying físico que con las de otras manifestaciones de bullying, en tanto que las mujeres empatizaron con todas las víctimas de bullying de manera similar.

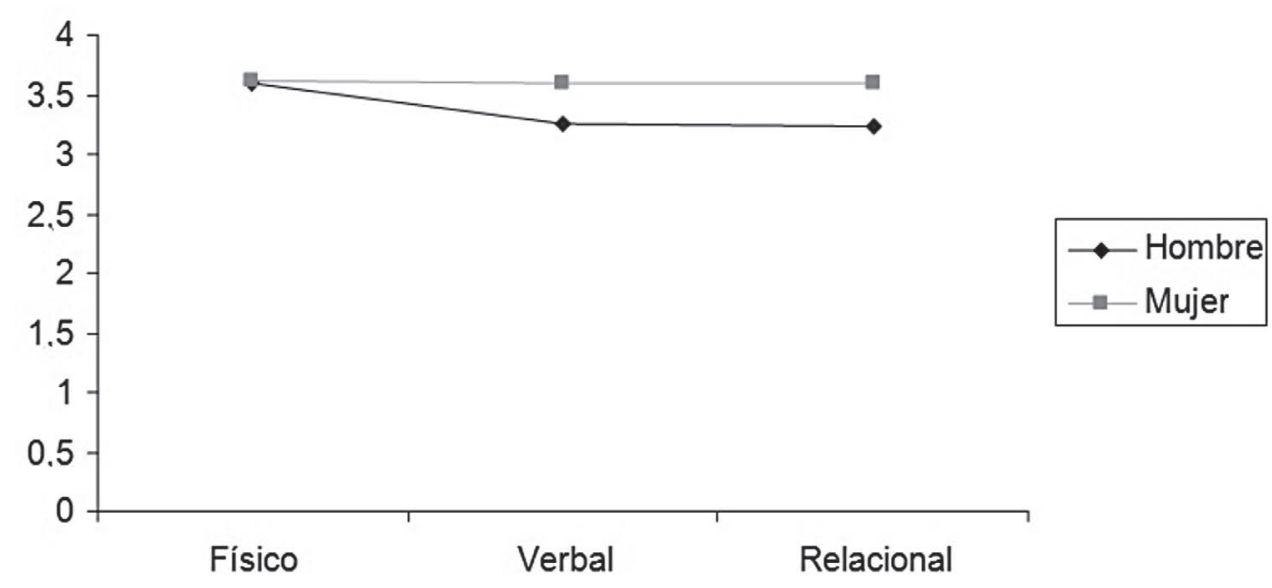

Figura 2. Medias de empatía con la víctima, según sexo y tipo de bullying.

Al analizar el efecto principal del tipo de bullying por pares, se encontró una diferencia significativa en los puntajes de empatía con la víctima entre el bullying físico y los otros dos tipos $(p<0,05)$, en tanto que no se encontró diferencia significativa $(p=0,90)$ entre el bullying verbal y relacional (ver Figura 3).

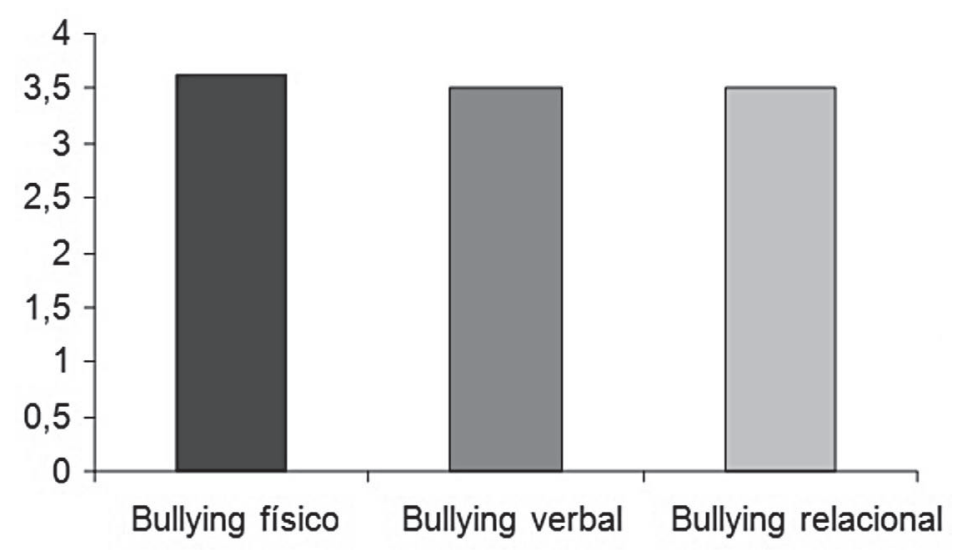

Figura 3. Medias de empatía con la víctima, según tipo de bullying. 
El ANOVA mixto también reveló un efecto principal del tipo de bullying en la variable disposición a intervenir, es decir, hubo diferencias significativas en la disposición a intervenir para cada tipo de bullying, $F=11,47, p<0,001, \eta^{2}=0,18,95 \%$ ICs $[4,89,4,99],[4,77,4,90]$ y $[4,61,4,82]$ para bullying físico, verbal y relacional, respectivamente. No se detectó un efecto significativo de la interacción con sexo, $F=1,32, p=0,268$.

Analizando las comparaciones por pares de los efectos principales del tipo de bullying, se encontraron diferencias significativas entre los puntajes de disposición a intervenir para cada tipo de bullying $(p<0,05)$, como puede verse en la Figura 4.

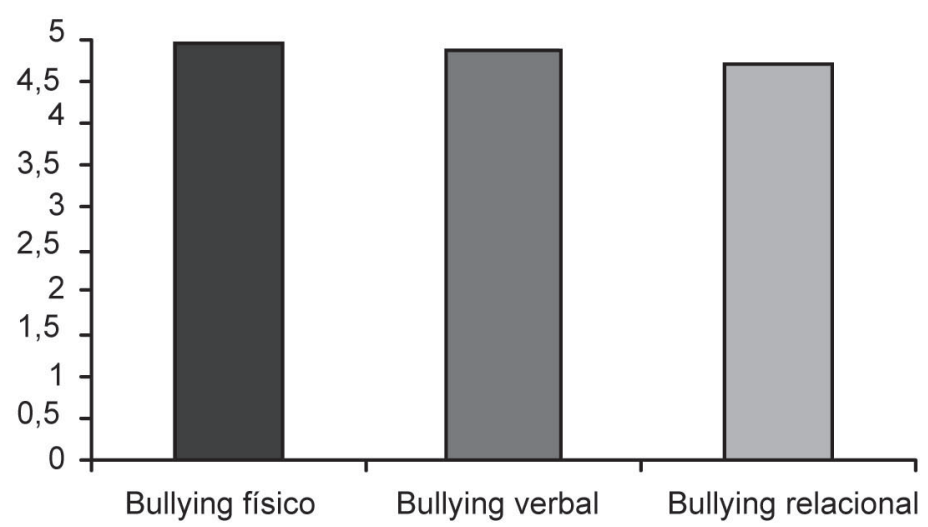

Figura 4. Medias de disposición a intervenir, según tipo de bullying.

\section{Discusión}

Los resultados obtenidos muestran que la percepción de gravedad, empatía y disposición a intervenir fue menor para el bullying relacional que para el bullying físico.

Los resultados generales indican que existiría la creencia entre los profesores de que el bullying físico es más grave que los otros tipos, mostrando más empatía con las víctimas y una mayor disposición a intervenir. Así, también, los análisis de efectos principales muestran que existiría una jerarquía percibida en los tipos de bullying, en la que el bullying físico es considerado más grave que el verbal, el cual, a su vez, es percibido como más grave que el relacional. Este patrón se repite en la variable disposición a intervenir, manifestando los profesores una mayor disposición a intervenir en situaciones de bullying físico que en situaciones de bullying verbal, dejando al bullying relacional en último lugar. No obstante, en esta variable se observó menor dispersión de los valores en bullying físico y verbal que en bullying relacional. Ello implicaría que, si bien los participantes puntuaron alto en su disposición a intervenir en todos los tipos de bullying, en el relacional existen mayores diferencias entre los profesores: algunos puntuaron alto pero otros puntuaron más bajo. En relación con la variable empatía con la víctima, si bien se mantiene la preponderancia del bullying físico, los bullying verbal y relacional son equivalentes.

Si bien estos resultados coinciden con los hallazgos de Yoon y Kerber (2003) y Bauman y Del Rio (2006), cabe señalar que en este estudio las diferencias de promedios encontradas, si bien fueron estadísticamente significativas, en general fueron mínimas. Asimismo, los puntajes obtenidos en cada una de las variables estudiadas fueron altos, lo cual, desde el punto de vista cualitativo, podría reflejar que no existen diferencias sustantivas entre los distintos tipos de bullying. Esto podría deberse a que en los últimos años y en el período en que se llevó a cabo este estudio el tema del bullying ha ocupado un importante espacio a nivel de los medios de 
comunicación y de las instituciones educativas. En esta línea y desde el modelo ecológico de Bronfenbrenner, los profesores estarían en interacción permanente con dichos mensajes, insertos e influidos por la cultura que actualmente identifica y reconoce el hostigamiento escolar como un problema social frente al que debe intervenirse. Pese a que los instrumentos de los profesores fueron respondidos en forma anónima y su participación fue voluntaria, no se controló el efecto de la deseabilidad social, lo que podría haber influido en que los profesores respondieran desde un deber normativo socialmente aceptable. No obstante lo anterior, aún se mantendría la tendencia de visibilizar con mayor fuerza la agresión física que la relacional y verbal.

Los hombres puntuaron más bajo que las mujeres en todas las variables estudiadas. Sin embargo, solo se encontró un efecto significativo de la interacción en la variable empatía con la víctima. El efecto encontrado muestra que los hombres y mujeres no difieren en su nivel de empatía hacia las víctimas de bullying físico; sin embargo, los hombres tienden a empatizar menos que las mujeres con las víctimas de los otros dos tipos de bullying. Esto es comprensible si se considera que la agresión está más "validada" en la cultura masculina y que la agresión relacional se da con mayor frecuencia en mujeres que en hombres (Veenstra et al., 2005), por lo cual las mujeres podrían ser más empáticas y estar más dispuestas a intervenir que los hombres. Asimismo, la agresión física y verbal pueden ser más comunes en una cultura masculina (Veenstra et al., 2005) y, por lo tanto, los profesores hombres le otorgarían menor gravedad, tendrían menor empatía y estarían menos dispuestos a intervenir.

Una limitación del estudio fue el tipo muestra, ya que esta no fue probabilística y los profesores pertenecían a colegios de un nivel socioeconómico medio-alto, lo cual limita el alcance de los resultados y queda la interrogante sobre lo que ocurre en otros niveles socioeconómicos de escuelas públicas y particulares subvencionadas, donde los valores y pautas culturales pueden tener otros matices. Sería necesario replicar este estudio con muestras de colegios de otros tipos de dependencia administrativa, con el fin de conocer cómo los profesores perciben y enfrentan los distintos tipos de bullying en otras realidades. Asimismo, en un próximo estudio debiera incorporase en el instrumento viñetas de cyber-bullying, ya que constituye una situación de acoso que se ha instalado en las escuelas y que en este estudio no se investigó.

Finalmente, surge la pregunta en relación al tipo de intervención y el rol que los profesores asumirían ante una situación de hostigamiento que, aun cuando no fue una interrogante de este estudio, sería relevante estudiarlo. Si bien es un paso importante que el bullying se identifique y se le otorgue gravedad, queda el desafío de identificar el tipo de intervenciones que deberían realizar los profesores con las víctimas y agresores en la dinámica grupal donde se dan dichas situaciones, más aún considerando que una de las razones por las que a veces los profesores no intervendrían sería por la falta de herramientas para hacerlo (Mishna et al., 2005). Futuros estudios deberían explorar ambos elementos.

\section{Referencias}

Alexander, C. S. \& Becker, H. J. (1978). The use of vignettes in survey research. Public Opinion Quarterly, 42, 93-104. doi:10.1086/268432

Batsche, G. M. (2002). Bullying. En G. G. Bear, K. M. Minke \& A. Thomas (Eds.), Children's needs II: Development, problems and alternatives (pp. 171-180). Bethesda, MD: National Association of School Psychologists.

Bauman, S. \& Del Rio, A. (2006). Preservice teachers' responses to bullying scenarios: Comparing physical, verbal, and relational bullying. Journal of Educational Psychology, 98, 219-231. doi:10.1037/0022-0663.98.1.219

Berger, C. \& Lisboa, C. (2009). Hacia una comprensión ecológica de la agresividad entre pares en el microsistema escolar. En C. Berger \& C. Lisboa (Eds.), Violencia escolar: estudios y posibilidades de intervención en Latinoamérica (pp. 58-81). Santiago, Chile: Editorial Universitaria.

Bonilla, S. \& Oviedo, P. (2011). El bullying desde la perspectiva de los docentes de colegios del sector oriente de Santiago (Tesis de Magíster no publicada), Facultad de Psicología, Universidad del Desarrollo, Santiago, Chile.

Boulton, M. J. (1997). Teachers' views on bullying: Definitions, attitudes and ability to cope. British Journal of Educational Psychology, 67, 223-233. doi:10.1111/j.2044-8279.1997.tb01239x

Bronfenbrenner, U. (1979). The ecology of human development: Experiments by nature and design. Cambridge, MA: Harvard University Press.

Card, N. A., Stucky, B. D., Sawalani, G. M. \& Little, T. D. (2008). Direct and indirect aggression during childhood and adolescence: A meta-analytic review of gender differences, intercorrelations and relations to maladjustment. Child Development, 79, 1185-1229. doi.10.1111/j.1467-8624.2008.01184x 
Cohn, A. \& Canter, A. (2002). Bullying: Facts for schools and parents. Bethesda, MD: National Association of School Psychologists. Extraído de http://www.naspcenter.org/factsheets/bullying_fs.html

Craig, W. M., Henderson, K. \& Murphy, J. G. (2000). Prospective teachers' attitudes towards bullying and victimization. School Psychology International, 21, 5-21. doi:10.1177/014303430021001

Crick, N. R. (1996). The role of overt aggression, relational aggression, and prosocial behavior in the prediction of children's future social adjustment. Child Development, 67, 2317-2327. doi:10.1111/j.1467-8624.1996.tb01859.x

Crick, N. R. \& Bigbee, M. A. (1998). Relational and overt forms of peer victimization: A multiiformant approach. Journal of Consulting and Clinical Psychology, 66, 337-347. doi:10.1037/0022-006x.66.2.337

Crick, N. R. \& Grotpeter, J. K. (1995). Relational aggression, gender, and social-psychological adjustment. Child Development, 66, 710-722. doi:10.1111/j.1467-8624.1995.tb00900.x

Doll, B., Song, S. \& Siemers, E. (2004). Classroom ecologies that support or discourage bullying. En D. L. Espelage \& S. M. Swearer (Eds.), Bullying in American schools: A social-ecological perspective on prevention and intervention (pp. 161-183). Mahwah, NJ: Lawrence Erlbaum.

Espelage, D. L., Mebane, S. E. \& Swearer, S. M. (2004). Gender differences in bullying: Moving beyond mean level differences. En D. L. Espelage \& S. M. Swearer (Eds.), Bullying in American schools: A social-ecological perspective on prevention and intervention (pp. 15-35). Mahwah, NJ: Lawrence Erlbaum.

Gould, D. (1996). Using vignettes to collect data for nursing research studies: How valid are the findings? Journal of Clinical Nursing, 5, 207-212. doi:10.1111/j.1365-2702.1996.tb00253.x

Kaltiala-Heino, R., Rimpelä, M., Rantanen, P. \& Rimpelä, A. (2000). Bullying at school—an indicator of adolescents at risk for mental disorders. Journal of Adolescence, 23, 661-674. doi:10.1006/jado.2000.0351

Lecannelier, F. (2007). Apego e intersubjetividad: influencia de los vínculos tempranos en el desarrollo humano y la salud mental. Santiago, Chile: Lom.

Leff, S. S., Kupersmidt, J. B., Patterson, C. J. \& Power, T. J. (1999). Factors influencing teacher identification of peer bullies and victims. School Psychology Review, 28, 505-517.

Magendzo, A., Toledo, M. I. \& Rosenfeld, C. (2004). Intimidación entre estudiantes. ¿Cómo identificarla? ¿Cómo atenderla? Santiago, Chile: Lom.

Mishna, F., Scarcello, I., Pepler, D. \& Wiener, J. (2005). Teachers' understanding of bullying. Canadian Journal of Education, 28, 718-738.

Newman, R. S. \& Murray, B. J. (2005). How students and teachers view the seriousness of peer harassment: When is it appropriate to seek help? Journal of Educational Psychology, 97, 347-365. doi:10.1037/0022-0663.973.347

Nicolaides, S., Toda, Y. \& Smith, P. K. (2002). Knowledge and attitudes about school bullying in trainee teachers. British Journal of Educational Psychology, 72, 105-118. doi:10.1348/000709902158793

Olweus, D. (1993). Bullying at school: What we know and what we can do. Cambridge, MA: Blackwell.

Olweus, D. (1995). Bullying or peer abuse at school: Facts and intervention. Current Directions in Psychological Science, 4, 196-200. doi:10.1111/1467-8721.ep10772640

Pérez, V. (2008). Comparación de la percepción de los profesores en relación a los tipos de bullying: físico, verbal y relacional (Tesis de Magíster no publicada), Facultad de Psicología, Universidad del Desarrollo, Santiago, Chile.

Pérez, V., Fernández, A. M., Rodríguez, J. \& De la Barra, F. (2005). Efectividad de una estrategia conductual para el manejo de la agresividad en escolares de enseñanza básica. Psykhe, 14(2), 55-62. doi:10.4067/S071822282005000200005

Poulou, M. (2001). The role of vignettes in the research of emotional and behavioural difficulties. Emotional and Behavioural Difficulties, 6, 50-62. doi:10.1080/13632750100507655

Sharp, S. (1995). How much does bullying hurt? The effects of bullying on the personal wellbeing and educational progress of secondary aged students. Educational and Child Psychology, 12(2), 81-88.

Smith, P. K. \& Brain, P. (2000). Bullying in schools: Lessons from two decades of research. Aggressive Behavior, 26, 1-9. doi:10.1002/(SICI)1098-2337(2000)

Sullivan, K., Cleary, M. \& Sullivan, G. (2003). Bullying en la enseñanza secundaria. El acoso escolar: cómo se presenta y cómo afrontarlo. Barcelona, España: Ediciones Ceac.

Veenstra, R., Lindenberg, S., Oldehinkel, A. J., De Winter, A. F., Verhulst, F. C. \& Ormmel, J. (2005). Bullying and victimization in elementary schools: A comparison of bullies, victims, bully/victims, and uninvolved preadolescents. Developmental Psychology, 41, 672-682. doi:10.1037/0012-1649.41.672

Wilks, T. (2004). The use of vignettes in qualitative research into social work values. Qualitative Social Work, 3, 78-87. doi:10.1177/1473325004041133

Yoon, J. S. \& Kerber, K. (2003). Bullying: Elementary teachers' attitudes and intervention strategies. Research in Education, 69, 27-34.

Zerón, A. M. (2006). Sentidos de la violencia escolar en Chile: un estudio sociológico (Tesis de Doctorado no publicada), Facultad de Educación, Pontifica Universidad Católica de Chile y Faculté de Sciences de l'Education de l’Université de Bordeaux, Santiago, Chile.

Fecha de recepción: Septiembre de 2009.

Fecha de aceptación: Octubre de 2011. 


\section{Anexo \\ Viñetas Modificadas}

A continuación se presenta una serie de situaciones de la vida escolar. Le pedimos que lea atentamente cada una de ellas y que responda las preguntas que se encuentran a continuación de cada situación. Además, le rogamos completar los datos de identificación que se señalan, sin poner su nombre.

Nivel:

Años que lleva trabajando en el Colegio

Sexo: Mujer..............

Hombre:

\section{Situación $\mathrm{N}^{\mathrm{o}} 1$}

Usted escucha a un estudiante cantarle a otro niño: "Mamón de la profesora, chupamedias". El niño trata de ignorar los comentarios, pero se queda molesto y callado en su escritorio. Usted ha presenciado esta interacción entre los mismos niños varias veces con anterioridad.

1. En su opinión, ¿cuán grave es esta situación?

1. No es nada grave

2. No muy grave

3. Moderadamente grave

4. Grave

5. Muy grave

2. Yo estaría enfadado por el comportamiento del alumno y me sentiría solidario con el niño.

1. Estoy en absoluto desacuerdo

2. No estoy de acuerdo

3. No estoy de acuerdo ni en desacuerdo

4. Estoy absolutamente de acuerdo

3. ¿Cuán probable es que usted intervenga en esta situación?

1. Es absolutamente improbable

2. No muy probable

3. Medianamente probable

4. Probable

5. Muy probable

4. Si usted responde frente a esta situación, ¿que haría con el agresor?

5. ¿Que haría usted con la víctima?

En las situaciones 2 a 6 se han eliminado las preguntas porque son iguales a las de la Situación 1.

\section{Situación $\mathrm{N}^{\circ} 2$}

Durante la hora de taller, usted escucha por casualidad a un niño decir a otro estudiante: "Si no me das el plumón rojo, no te invito a mi fiesta". Esta no es la primera vez que usted escucha a este niño decirle este tipo de cosas a este compañero. 


\section{Situación $\mathrm{N}^{\mathrm{o}} 3$}

Su clase se está preparando para el almuerzo y los niños están en fila frente a la puerta. Usted escucha a un niño manifestar a otro: “¡Oye, dame la plata de tu almuerzo!”. El niño no le hace caso pero el compañero le empieza a cantar: "Hijito de mamá, gay... mariquita". No es primera vez que usted observa que este niño le canta esta canción a su compañero.

\section{Situación $\mathrm{N}^{\circ} 4$}

Un alumno trajo al colegio un MP3. Cuenta que este fue un premio proveniente de una rifa. Otro niño se acerca y golpea su cabeza reclamando el MP3. Inicialmente el niño rehúsa, pero finalmente cede. Esta tipo de interacción ha ocurrido varias veces anteriormente entre estos niños.

\section{Situación $\mathbf{N}^{\circ} 5$}

Usted ha permitido que los niños de su clase tengan un pequeño tiempo libre debido a que hoy día han trabajado muy duro. Usted presencia que un niño le dice a otro: "Ya te he dicho que no puedes jugar con nosotros". El niño juega solo durante el tiempo restante con lágrimas en sus ojos. Esta no es la primera vez que este niño ha excluido al otro del juego.

\section{Situación $\mathrm{N}^{\circ} 6$}

Usted ha asignado a los niños de su clase para que trabajen en grupos de 4 para hacer proyectos. Mientras que los niños están reuniéndose en sus grupos, usted ve a un alumno empujar a otro niño con tanta fuerza que este último cae al suelo. El empujón fue claramente intencional y no fue resultado de una provocación. El niño que cayó grita: “ĐDeja de empujarme. Siempre me haces esto, por favor, ándate, déjame tranquilo!" 